\title{
Automated Liquid-Level Monitoring and Control using Computer Vision
}

\author{
Tara Zepel, ${ }^{1}$ Veronica Lai, ${ }^{1}$ Lars P.E. Yunker, ${ }^{1}$ and Jason E. Hein ${ }^{1}$ \\ 1 The University of British Columbia, Department of Chemistry, Vancouver, BC V6T 1Z1, Canada. \\ tarazee@gmail.com, veronica.lai.vn@gmail.com,larsy@chem.ubc.ca,jhein@chem.ubc.ca
}

\begin{abstract}
Chemists spend an inordinate amount of time performing low-level tasks based on visual observation. Camera-enabled laboratory equipment in conversation with computer vision algorithms can be used to automate many of these processes, thereby freeing up valuable time and resources. We developed a generalizable computer-vision based system capable of monitoring and controlling liquid-level across a variety of chemistry applications. This paper reports on the system's motivation, architecture, and successful deployment in three experimental use cases which require continous stirring: continuous preferential crystallization (CPC), slurry filtration, and solvent swap distillation.
\end{abstract}

\section{Introduction}

Chemists spend an inordinate amount of time performing low-level tasks based on visual observation. One such task is liquid-level recognition. Monitoring and being able to control the location of a liquid-air or liquid-liquid interface is a crucial factor in a wide variety of common laboratory procedures.1,2 Processes that involve the addition, extraction or transfer of liquid can fail or result in dangerous conditions if the total volume or phase-boundary extends beyond a specified tolerance zone. At the industrial-scale, maintenance of liquidlevel within this zone can be facilitated by sensors or large allowable margins of error,,$^{3-5}$ At the smaller-scale, such accommodations are infeasible. Detection and control of liquidlevel are often tasked to the researcher, who must visually monitor the experiment and make any adjustments they deem necessary. This is an easy task for a human researcher, but one that is often tedious and time consuming.

As laboratories become increasingly smart and increasingly connected, so does our ability to automate discovery and experimentation. Computer vision can be used to assess and control experimental procedures that typically rely on visual feedback. Digital imagining technology in conversation with computer vision algorithms has been used to monitor sample quality, detect physical properties (e.g. color, crystal size, etc.), and perform in situ analysis. ${ }^{6-9}$ When combined with smart machinery and a clear set of instructions, computer vision can also be used to drive control decisions.

Our group has focused on bringing together robotics and data-rich monitoring techniques to develop autonomous platforms that drive discovery and reaction optimization. Images provide yet another data-rich source that can be used to gather and analyze information. Recently, we encountered a situation in our lab that led us to develop HeinSight, an inexpensive and generalizable computer vision system for liquid-level monitoring and control (Figure 1). It consists of three main components: a pump, a webcam, and series of custom Python scripts. Compared with similar systems, which rely on the addition of a mechanical ${ }^{10,11}$ or density-based sensors, ${ }^{3,6,12-15}$ our system is minimally invasive and easy to use. Its modular design enables customization and it is easily integrated into a variety of existing experimental set-ups (Figure 1). The addition of a graphical user interface (GUI) and Slack integration greatly lower the barrier to use. The entire system can be set up, controlled, and remotely monitored without the manipulation of code. This paper reports on the system's motivation, development, and successful deployment across three experimental use cases: continuous preferential crystallization (CPC), continuous filtration, and evaporative solvent swap.

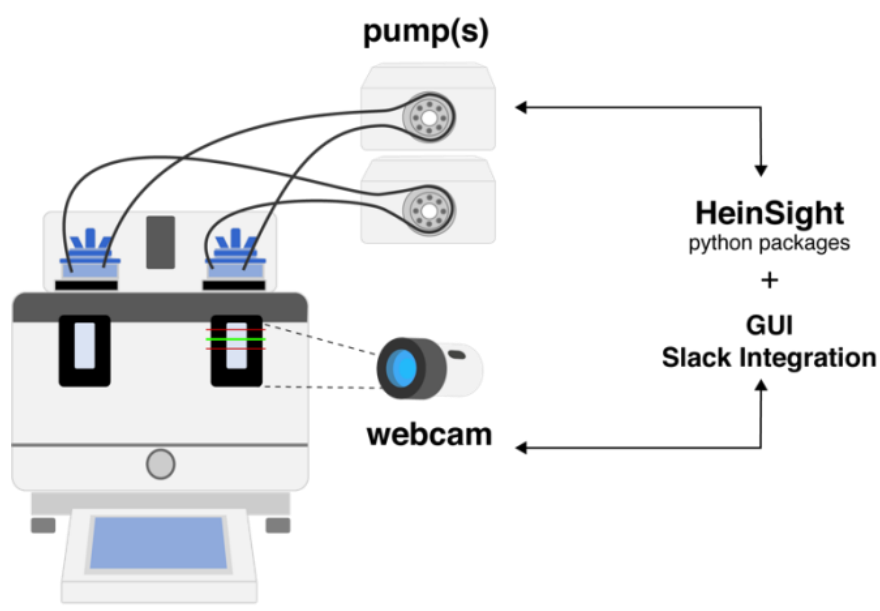

\section{Exp. Set-up}

Figure 1. Example illustration of HeinSight being used to monitor and control liquid-level in a dual-pump CPC experiment conducted in a Mettler Toledo EasyMax. The user interacts with the system through a GUI and Slack integration on the front end. Pump and webcam behaviour is controlled by custom python packages on the backend.

\section{Motivation}

Initial motivation for the system came from a recurrent problem encountered in our lab when trying to automate continuous preferential crystallization (CPC) experiments. CPC involves the 
continuous transfer of liquid between two (or more) vessels in the presence of a seed to preferentially crystallize out a desired compound. 7,8 This is a time-intensive physical process, often requiring runs of $48+$ hours. Previous work in our lab had developed automation capabilities for liquid transfer between vessels, sampling, and reaction monitoring. However, the distribution of liquid in the system still required periodic checking and manipulation. If the liquid-level became too unbalanced between the two receptacles, the experiment would need to be reset or adjusted to account for current conditions. In extreme cases, too much liquid would transfer into a single vessel and the system would overflow. To avoid either of the above scenarios, researchers found themselves having to check-in on the system every few hours. The need to manually monitor and adjust a running experiment greatly hinders researchers' productivity. It is especially challenging for experiments that require long run times, such as CPC. Even far simpler processes, such as filtering a large volume of solution through a small-volume apparatus, can drain researcher time and resources.

\section{Previous Work}

We began looking for solution to recognizing materials and phase-boundaries inside reaction vessels and quickly realized the need for a generalizable, easy-to-use, and inexpensive computer vision-based system. Eppel and Kachman have identified algorithms that are able to determine liquid-liquid or liquid-air interfaces in transparent vessels of varying shapes. ${ }^{1,2}$ Recent expansion into the area of machine learning has shown improved accuracy, particularly when using neural nets. ${ }^{17-19}$ Unfortunately, we did not have a database of images at our disposal and wanted to direct our efforts at our area of expertise - building a fully integrated autonomous monitoring and control system. Ley and co-workers have developed a series of continuous-flow liquid-liquid extraction systems that determine the location of the interface by monitoring an added green float. ${ }^{17,18}$ While ideal in certain scenarios, we were not able to adopt their approach. Our applications require continuous stirring and monitoring any additive object would be complicated by lateral or erratic shifting due to the presence of a vortex. Additionally, we wanted to design a system that did not require additional probes or equipment and could be used across a variety of experimental set-ups, regardless of chemical composition. The system should be as flexible as possible and capable of supporting variation in solution density and color as well as shape and volume of reaction container.

\section{Materials and Methods}

\section{System Architecture}

Heinsight provides automated monitoring and control of liquidlevel within a user defined area across a variety of common continuously stirred tank reactor (CSTR) processes. The composite system comprised of a webcam, a series of customdeveloped Python packages, and peristaltic pump(s) (Figure 2) is designed to be modular and easily integrated into any benchtop setup at minimal cost. Currently supported experimental types include 1) single pump CPC, 2) dual pump CPC, 3) continuous filtration, and 4) evaporative solvent swap.

By creating different Python modules for pump control, computer vision, and overall system control, we have left the option open for researchers to adapt our code to their preferred hardware and experimental workflow. We chose to use a Logitech C922x webcam (720p at 60fps) and New Era peristaltic pumps (NE-9000) as hardware as they are both readily available and the latter has well-documented serial protocols. $^{3}$ In practice, any USB webcam and pump capable of receiving serial commands may be used. The packages rely on open-source libraries (Numpy, OpenCV, and PySerial) so that there is no financial barrier to usage or adaptations. For additional ease of use, we wrapped the code in a GUI and incorporated Slack bot user integration. The system can be fully customized and run from the GUI while integration with the Slack messaging service provides remote online monitoring and control.

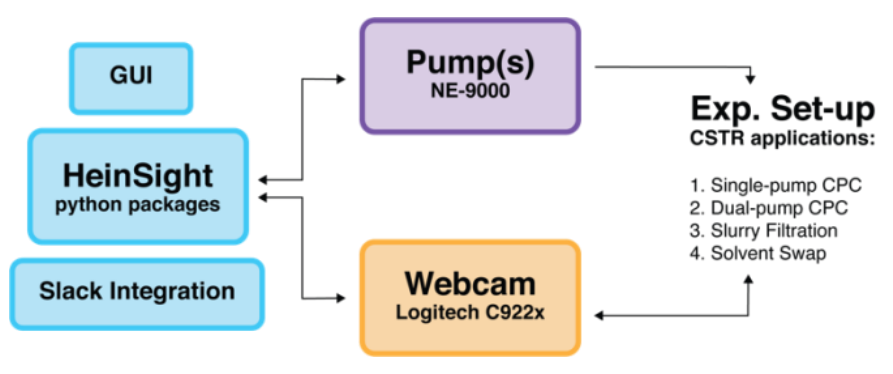

Figure 2. Schematic view of system components and relations.

\section{Control Logic}

Automated control of liquid-level follows the same logic regardless of experimental type. Once triggered, the automation loop (Figure 3) proceeds through the following steps, where $n$ and $s$ are user-defined parameters set prior to each run.

1. Start default pump behavior.

2. Wait $n$ seconds.

3. Monitor liquid-level (capture images + apply liquidlevel finding algorithm).

4. Determine if level is within tolerance.

a. If yes, return to step 2 .

b. If no, determine if liquid needs to be withdrawn of dispensed and pump in appropriate for $s$ seconds. Return to step 3 . 


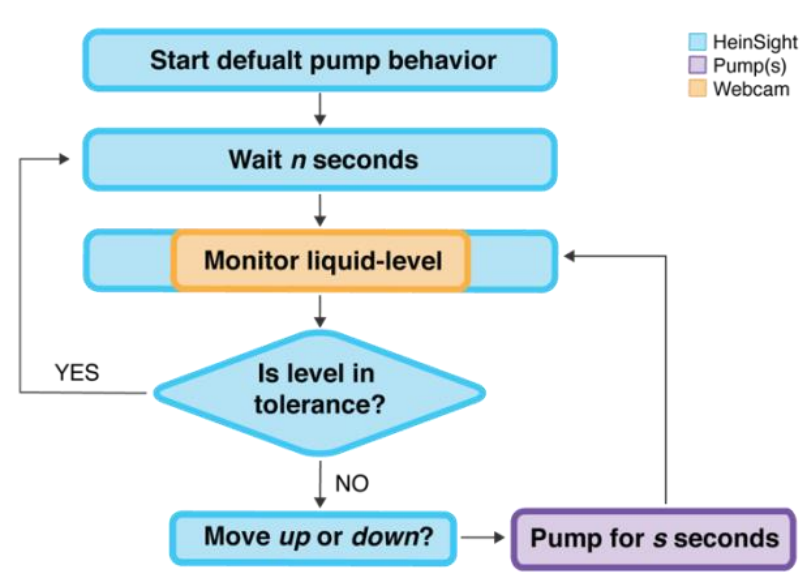

Figure 3. HeinSight control loop. Steps are color-coded to match the component performing the action in correlation with Figure 2

The system will cycle through this process, starting, stopping, and adjusting pump parameters until instructed to stop or a critical error occurs. For example, if the liquid-level is determined to be too high, the system will trigger the pump to withdraw from the watched vessel for $s$ seconds. The liquidlevel will be monitored and assessed again and the loop continues. While the control logic operates consistently across experimental types, the different processes require slightly different control parameters. For example, when performing CPC (or any other experiment that requires bi-directional liquid exchange), the location of liquid-level can drift in either of two directions, above or below a user-set reference line (see the following section). However, when performing a filtration or solvent swap experiment, the location of the liquid level almost always moves in a single direction. Once one of the preprogrammed experimental types has been selected in the GUI, it guides the user through the necessary configuration.

\section{Selecting a ROI, Reference, and Tolerance Zones}

Setting up an experiment in the GUI also prompts the user to define a series of visual boundaries that the system will use to make control decisions. The first, selecting a region of interest (ROI), serves two purposes: 1) it specifies the area which the liquid-level finding algorithm will search, and 2) it allows the user to exclude any areas that the algorithm might find confusing (e.g. tubing, glare, etc.). The purpose of this exercise is the removal of uninformative or unwanted visual information, thereby increasing efficiency and the likelihood of an accurate reading.

The second visual boundary the user is asked to define is a reference line, which marks the desired height of the meniscus. The only requirement is that it must be within the ROI. However, the system will perform better if the reference line is set in a way that avoids occlusions such as tubing or markings on the reaction container.
The third visual boundary defines a tolerance zone that the liquid-level must remain within. This is the zone in which the liquid-level location is programmed to remain. If the level drifts outside these bounds, self-correction be triggered. Different experimental types require differing methods of defining tolerance due to the direction in which the liquid-level is likely drift. For CPC experiments, the level can drift up or down. The user is thus prompted to select both upper and lower tolerance bounds. Solvent swap and filtration experiments generally result in the liquid-level drifting in a single direction (usually down), and the user is only asked to select one tolerance bound. For CPC runs, there is an additional option to set fail-safe tolerance levels. Should the liquid-level move outside the specified fail-safe zone, pumping will cease, and the Heinsightcontrolled components of the system will suspend operation.

Once set, the system uses the selected boundaries to make control decisions given the context of the selected experiment. We have implemented an intuitive way for the researcher to monitor what the code interprets as the run progresses. Each time an experiment is run using HeinSight, the system creates three folders of saved images:

1. raw_images: all images captured by the webcam

2. all_drawn_images: images overlaid with user-set boundaries

3. slack_images: images sent through the Slack messenger

Figure 4 shows an example image saved to the 'all_drawn_images' folder during a dual-pump CPC run. All boundaries defined by the user are shown along with location of the current liquid-level as determined by the liquid-level finding algorithm.

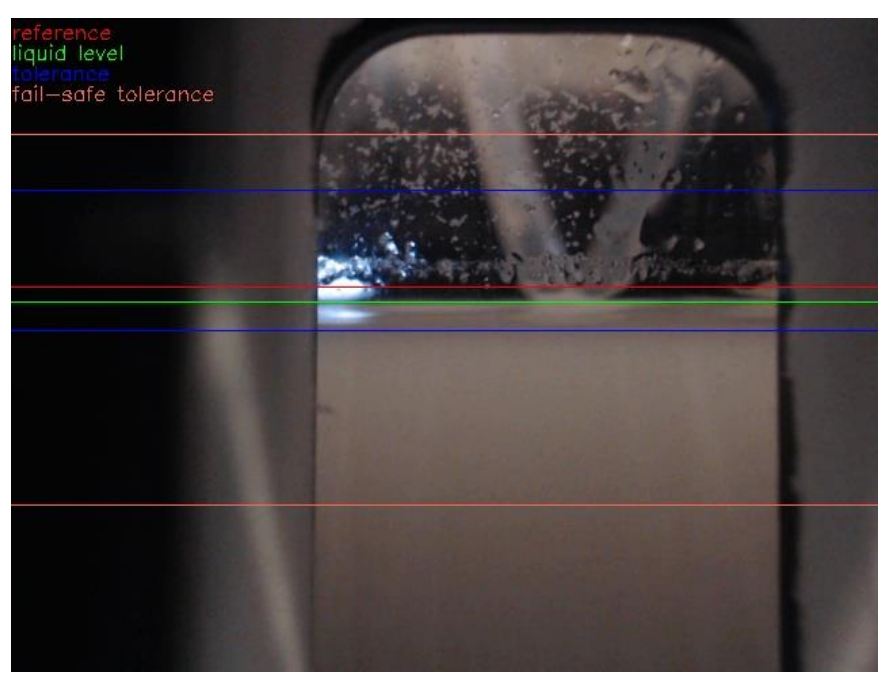

Figure 4. A 'drawn image' from a dual-pump CPC experiment. Color-coded lines are drawn at user defined locations ( red $=$ reference, green = current liquid-level, blue = tolerance bounds, pink = fail-safe tolerance bounds). 
Reviewing the 'all_drawn_images' folder allows the researcher to visually monitor how the liquid-level fluctuates in relation to defined boundaries in near real time. Images are saved as soon as current liquid-level location is determined and can also be combined to produce a video of the experiment once the run has completed (the Heinsight Python package includes a script which creates such a video). Review of the video by the researcher can be particularly helpful should the run require repetition or adjustment and offers the additional benefit of a record of visual changes in physical characteristics (e.g. crystal aggregation, turbidity, etc.).

\section{Monitoring Liquid-level}

The liquid-level detection algorithm we developed can locate the height of a liquid-air or liquid-liquid interface relative to the pixel height of an image. The computer-vision approach we have taken to accomplish to this is to find the strongest horizontal line, where strength is defined by contrast to surrounding vertical pixels. Following initial pre-processing (Figure 5a), the image is passed through a Canny edge detection algorithm, which outputs a binary image where strong edges are shown as white contours. ${ }^{20}$ Additional processing removes noise and vertical contours (Figure $\mathbf{5 b}$ ). The algorithm assumes that any interface boundary will be read as a relatively long horizontal line ( $\mathrm{ROI}$ selection is critically important here as additional horizontal lines confound the algorithm). The algorithm then divides the resulting image into horizontal slices 4 pixels high. Each slice is assigned a score based relative fraction of white pixels. The slice with the highest score within the user-defined ROI are identified as containing the liquidlevel, and the average vertical height the of pixels in the slice relative to the entire image is recorded as the current liquidlevel location (Figure 5c). All values output by the algorithm are saved to a JSON file for future analysis and review. Additional details and a step-by-step explanation of the algorithm are available in the Supporting Information.

Computer vision has a tendency to be finicky, and obtaining an accurate location measurement depends on a variety of environmental factors, most importantly lighting. Tubing, glare, residue stuck to the side of the reaction vessel or any external markings among other factors have the potential to confuse the algorithm. While we were able to optimize the visual environment across experimental set-ups to the best of our ability, errors still occasionally arose.

Consistency in the location measurement was improved allowing the user to specify whether the algorithm looks for the top or bottom edge of the meniscus. To account for the occasional error or incorrect identification due to unforeseen circumstances (e.g. a researcher walking in front of the camera), the system calculates an average relative liquid-level location across consecutive images. The number of images to be included in each average as well as the interval between checks are set by the user prior to the start of the experiment. For example, the user may instruct the system to take 5 consecutive images every 45 seconds. The system will determine the current liquid-level location in each of the 5 images and discard extreme outliers. Outliers are determined by applying a threshold to the modified z-score calculated for the liquid-level in each of the 5 images. Any level that deviates too far from the median is assumed to be invalid. The final number used to make the control decision as to whether the liquid-level is within tolerance is the average of the retained set.

a)

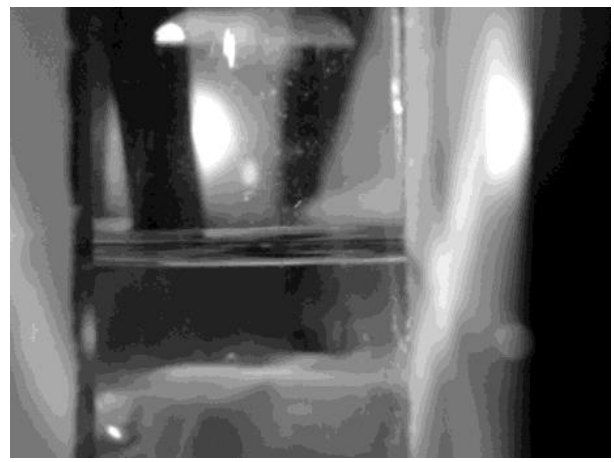

b)

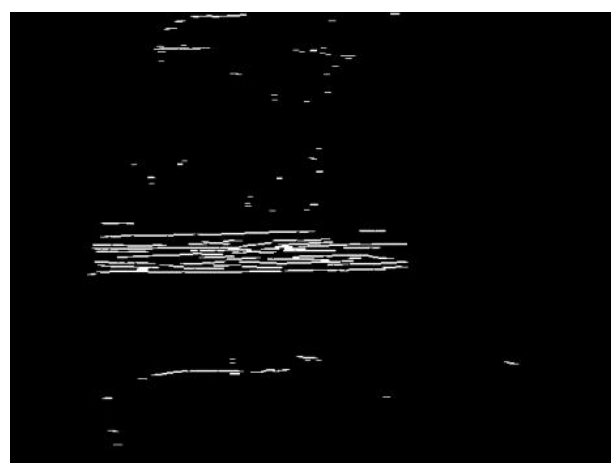

c)

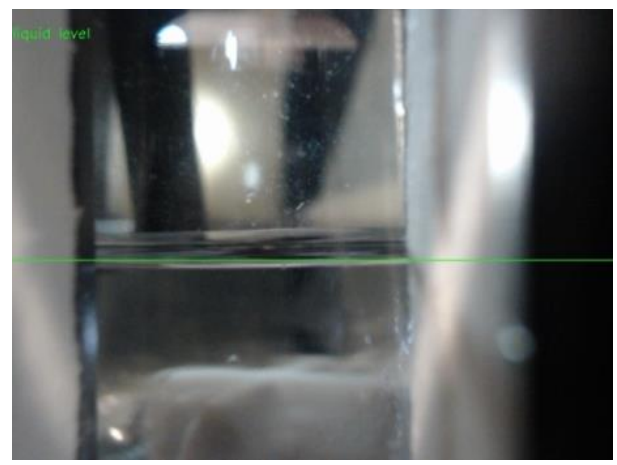

Figure 5a. Image after conversion to grayscale and CLAHE histogram normalization; $5 \mathbf{b}$. Binary image with vertical contours removed; $\mathbf{5 c}$. Original image with green line at current liquid-level location.

\section{Graphical User Interface}

The HeinSight system is designed to be generally applicable and easily incorporated into any laboratory. We are conscious of the fact that many existing automated systems are prohibitively expensive or require expert use. To make the system more accessible, we developed a graphical user interface. Users can adjust parameters and execute 
experiments entirely within the GUI without having to interface with any code.

The GUI was designed using PyQt5 and is configured to walk the user through specifying variables and performing necessary steps specific to the designated experimental type. Figure 6 shows the introductory screen upon launching the application. By default, the 'Set-Up' tab is active. The user is asked to select the experiment type and specify certain parameters related to configuration and control such as pump rate, the amount of time to wait between liquid-level monitoring and duration of self-correction. It is also possible to load parameters from a previously saved experiment and provide details for Slack integration. Only those variables relevant to the selected experimental type can be modified by users. For example, the option to enter a value for 'Pump 2 port' is disabled in Figure 6 because 'Single pump CPC' only uses one pump.

\begin{tabular}{|c|c|c|c|}
\hline \multicolumn{2}{|l|}{ Heinsight } & - & $\times$ \\
\hline \multicolumn{4}{|l|}{ File About } \\
\hline \multicolumn{4}{|l|}{ HeinSight } \\
\hline \multicolumn{4}{|c|}{ Automated Liquid Level Control } \\
\hline \multicolumn{4}{|l|}{ For Chemistry Applications } \\
\hline \begin{tabular}{l|l} 
Set-Up & Run \\
\end{tabular} & & & \\
\hline \multicolumn{3}{|l|}{ General } & - \\
\hline \multicolumn{2}{|l|}{ Experiment directory } & Browse... & \\
\hline \multicolumn{4}{|l|}{ Experiment name } \\
\hline Experiment type & Single pump CPC & & \\
\hline \multicolumn{4}{|l|}{ Peripherals } \\
\hline Camera port & 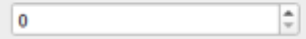 & & - \\
\hline \multicolumn{4}{|l|}{ Pump 1 port } \\
\hline \multicolumn{4}{|l|}{ Pump 2 port } \\
\hline Pump rate (mL/min) & $=$ & & \\
\hline Slack integration JSON & & Browse... & \\
\hline System Specifics & & & - \\
\hline
\end{tabular}

Figure 6. Screen shot of the 'Set-up' tab. Parameters can be entered by the user and saved to a .json file, which can be loaded to automatically populate fields in subsequent experiments.

The 'Run' tab (Figure 7) guides users through any pre-steps necessary for the application to accurately and dynamically control liquid-level given the previously set parameters. There are also options to preview the webcam livestream and initiate pumping to adjust the liquid-level prior to running the experiment. These steps help ensure that the liquid-level algorithm will perform adequately in the current physical environment. Automation begins when the user presses the 'Start experiment' button, prompting pop-up windows for ROI and tolerance zone selection. Once started, the system will run indefinitely until terminated by the researcher or encountering fail-safe conditions.

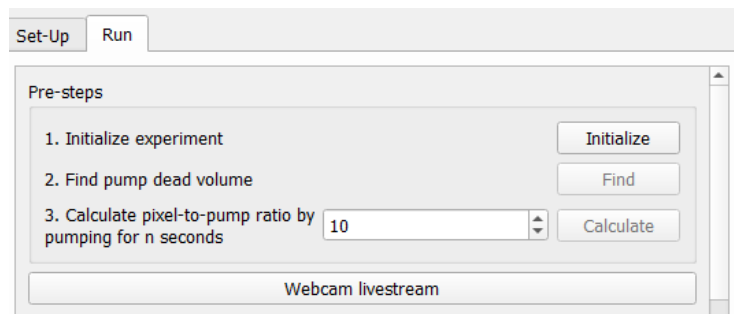

Figure 7. Screen shot showing the top half of the 'Run' tab. The Pre-Steps section walks the user through the actions that must be completed prior to starting a run of the selected experimental type.

\section{Remote Monitoring and Control}

Automation often comes with the corollary desire for remote monitoring and control. Researchers want to check-in on an experiment and know when key decisions are made. Given unsafe conditions, it is important that the experiment can be changed or shut down. We enabled communication between the user and the system during an active experiment using Slack, a popular online messaging platform. During set-up, the user is given the option to specify an existing Slack bot and channel to be used by the system. The specified bot will then send regular updates to the channel once an experiment has been started. We found it immensely helpful to know when the liquid-level moved outside tolerance bounds or if the system could not find a liquid-level. Figure $\mathbf{8}$ is a screenshot of the message the user received when the systems detects a need for self-correction.

gronckle APP 12:01 AM

application has been running for 6.51 hours and is still running

The last image taken

The last image taken -

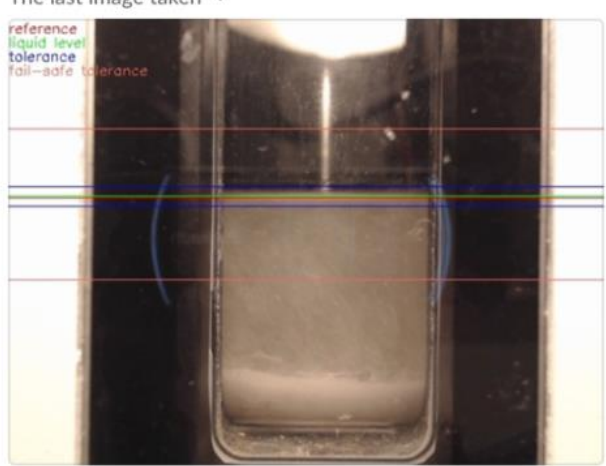

gronckle APP 12:09 AM

Liquid level moved above the tolerance boundaries. Current liquid level differs from the reference liquid level by 0.025833333333333364

to self correct need to withdraw. percent diff is: 0.025833333333333364 for 45

Figure 8. Slack messages sent by our bot, gronkle, during a dual-pump CPC experiment. The message shown here is sent when the system detects a need for self-correction and includes the image and measurements that resulted in this decision. Information is also provided about how the system will proceed. 
The Slack bot we created during development will also translate commands for action to the appropriate component of the HeinSight system. Users can remotely control an experiment by messaging a pre-programmed command: Hello, pause experiment, resume experiment, end experiment, current image, dispense, withdraw, liquid level graph [sec // min // hour]), and $A B O R T$. See the SI for further details on supported commands.

\section{Discussion and Results}

We tested and demonstrated the prototype system by conducting experiments in our lab in accordance with current projects and research. Emphasis was placed on proof of concept rather than novel chemistry. Successful applications of each of the pre-programmed experimental types are summarized below.

\section{Case 1: Continuous Filtration}

In the first use case, the system was deployed to orchestrate a filtration of a large volume of slurry through a filter with limited volumetric capacity. When faced with this issue, a researcher must typically spend an exorbitant amount of time progressively filling and refilling the filter as the slurry is gradually processed. If the slurry has particularly poor filtration properties (filter occlusion due to fine particles) this process can take days, as the researcher will typically only return intermittently to tend to the apparatus. In addition, this situation is particularly difficult to automate, as the rate of filtration will vary as the filter cake compresses over time, making a timed addition of the slurry inappropriate.

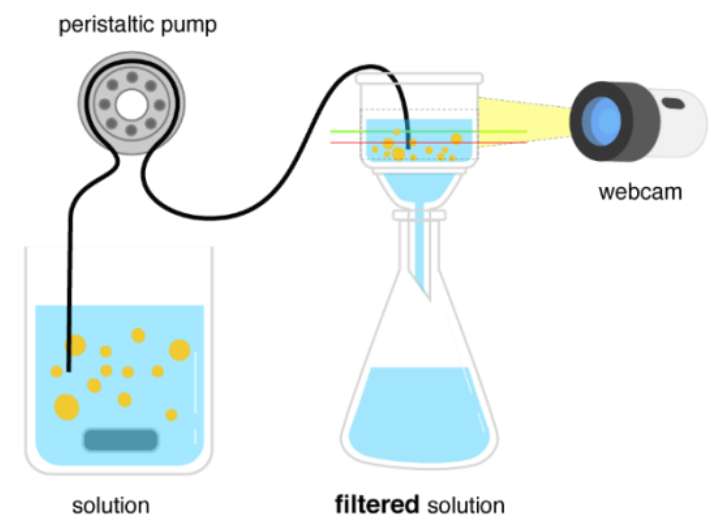

Figure 9. Experimental set-up for a continuous filtration run.

Successful deployment of the filtration application was demonstrated by passing a slurry of $0.995 \mathrm{~g}$ Celite and $\sim 250 \mathrm{~mL}$ $\mathrm{MeOH}$ through a gravity filtration set-up. The camera was positioned to watch the material as it passed into a funnel packed with glass wool (Figure 9). We found that a solid-colored background enabled the algorithm to "see" better by distinguishing the filtration set-up from the surrounding benchtop environment (see Figure S5b in "Continuous Filtration" section of the SI). HeinSight automatically replenished the slurry every time the meniscus dropped below the user-set level, thereby enabling continuous filtration. Data from the run is shown in Figure 10.

Filtration EX (Liquid-Level over Time)
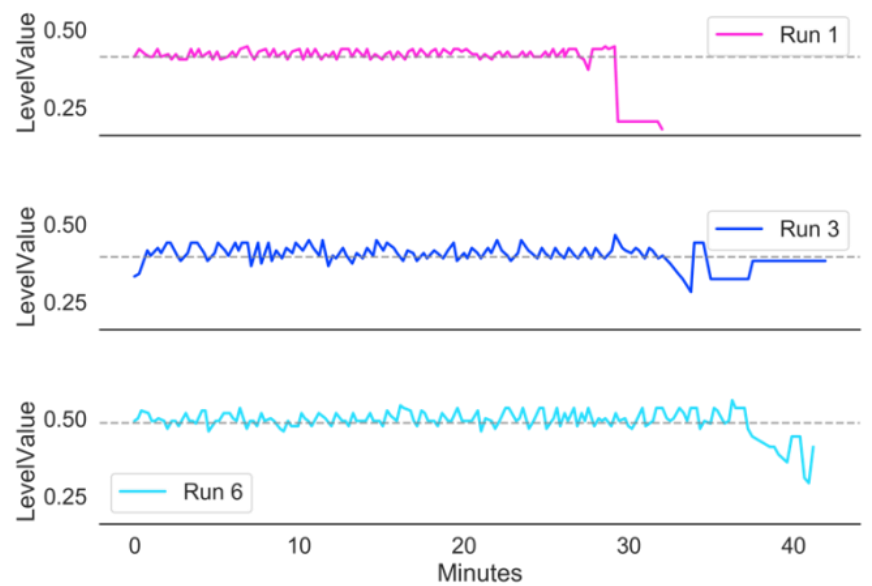

Figure 10. Relative liquid-level height over time during continuous filtration of Celite and $\mathrm{MeOH}$ slurry; grey dotted lines correspond to run specific tolerance level.

Eliminating the need for a human to monitor and dispense additional solution is most beneficial when dealing with large working volumes or substances that are particularly slow to filter. In theory, the HeinSight system can be used to automate the filtration of any volume using any method. To stress test the system, we set up an 'infinite' loop in which the slurry reservoir was also the collecting container. The system ran for 3 hours under these conditions.

\section{Case 2: Solvent Swap/Distillation}

When executing a synthetic chemical sequence, one reaction mixture may be held in the vessel while the solvent is distilled off and a new solvent is continuously added. ${ }^{21,22}$ This process, known as a solvent exchange distillation or a "solvent swap", allows multiple sequential chemical transformations to be executed on a reaction mixture, where very different solvent properties are required for each transformation. While this process is commonly executed on large scale, careful calculations must be completed to ensure the rate of fresh solvent addition and effluent distillation are balanced. The process is made more challenging, as physical properties of the solvent mix, such as the boiling point, will constantly change throughout the operation. The process could be made far simpler by instructing the pump to maintain a set liquid level while the distillation proceeds.

To demonstrate HeinSight's solvent swapping application, we used the system to automate the exchange of ethanol for toluene. The camera was positioned to watch the reaction 
vessel in a benchtop-scale distillation set-up (Figure 11). For ease of use, we placed the vessel in an EasyMax to ensure continuous stirring and temperature regulation. Approximately $250 \mathrm{~mL}$ of a $4: 1 \mathrm{EtOH}$ :toluene starting solution was heated to just above the boiling point of ethanol $\left(82^{\circ} \mathrm{C}\right)$, in this case the solvent with the lower boiling point. As ethanol was distilled out, an equivalent volume of Toluene was pumped in. The system ran successfully across multiple trials and was also used to automate the replenishment of starting solution in the purifications of DCM. Data from the EtOH:toluene swap is shown in Figure 12

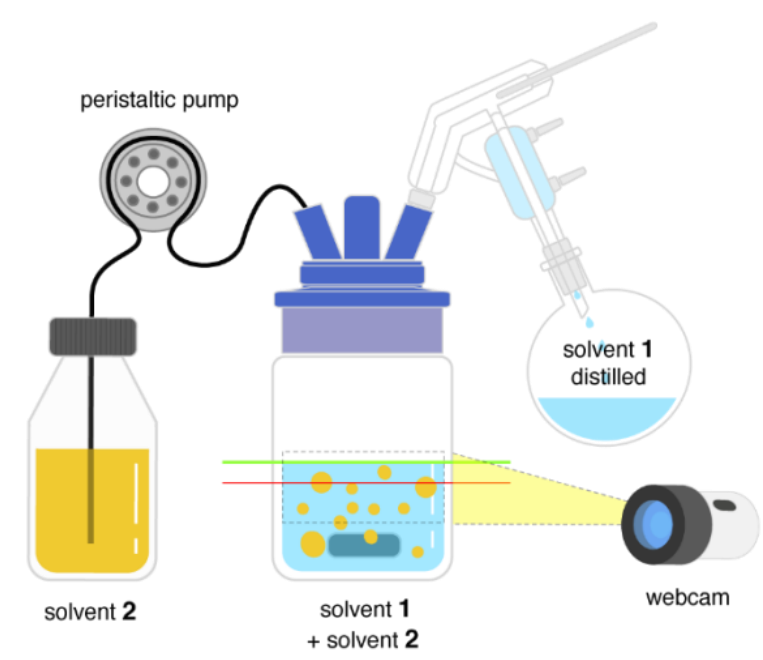

Figure 11. Experimental set-up for an evaporative solvent swap run.

Solvent Swap EX (Liquid Level over Time)
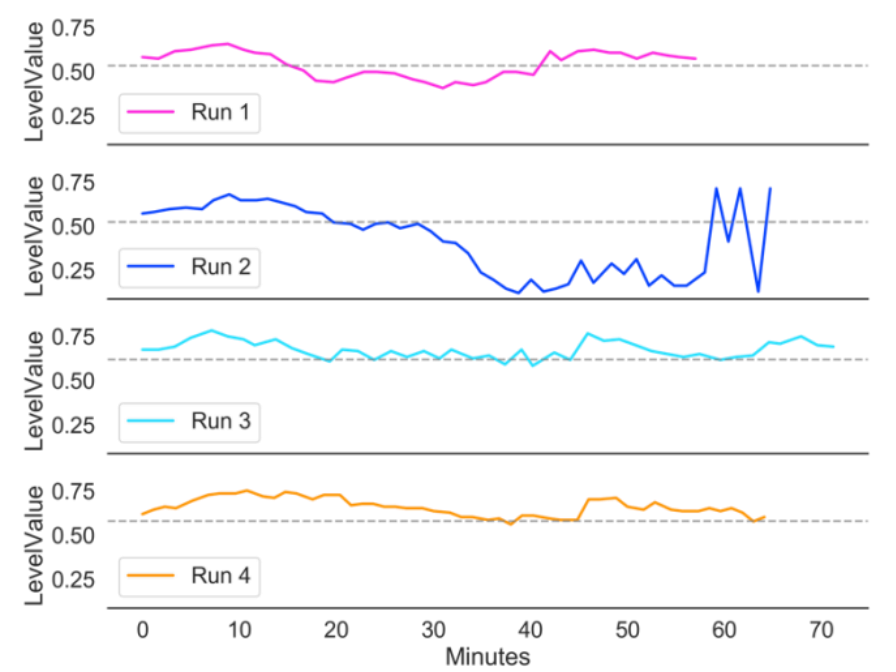

Figure 12. Relative liquid-level height over time during evaporative solvent swap of EtOH for toluene; every $10^{\text {th }}$ measurement shown. Grey dotted lines correspond run specific tolerance level.

Interestingly, the algorithm was able locate the meniscus even when monitoring the clear, uniform-color solution (see
Figure S10 in "Evaporative Solvent Swap" section of the SI). The backlight of the EasyMax was switched off so that only ambient lighting was used and automation continued unaffected even under conditions that would have been difficult for a human to monitor.

\section{Case 3: Continuous Preferential Crystallization}

Preferential crystallization (PC) is the general process by which a single enantiomer is isolated from a racemic mixture. ${ }^{23,24}$ This technique is can be accomplished when a metastable homogenous racemic mixture is seeded with crystals of the desired pure enantiomer, allowing selective crystallization via secondary nucleation. However, preferential crystallization may only be successful over limited timeframes, as the unseeded counter enantiomer remains supersaturated during the process and may undergo uncontrolled primary nucleation. One method of reducing the risk of uncontrolled nucleation is to limit the degree of supersaturation and maintain a near equilibrium solid/solution condition, but this will also limit the amount of material that can be isolated via typical batch crystallization.

In order to obtain high mass-throughput as well as limit unwanted crystallization the process can be executed via two coupled flasks which are held at different temperatures. The crude slurry is placed in one flask (dissolver) while the crystalfree solution phase is circulated between the two. The second flask (crystallizer) is seeded and held at a lower temperature than the dissolver, creating a mild supersaturation and allowing selective crystallization of the desired enantiomer. ${ }^{16,25}$ While similar processes have been adopted for numerous chemical manufacturing and large-scale applications, reducing to smaller bench-top applications with low working volumes is less common The primary challenge is again one of liquid level control; to operate at peak efficiency the crystal-free solution phase must be continuously circulated between dissolver and crystallizer via in line filters. As the process evolves, crystal growth and dissolution in each environment will alter the pumping efficiency, leading to sample overflow or interruption in the fluid circulation. To maintain control, the system must continually adapt, modifying and updating the pumping to maintain a balanced liquid level between the two flasks.

As we are manipulating a metastable fluid, approaches which use pulsed gas or level control by limited uptake can promote unwanted nucleation, resulting in batch failure. Furthermore, adding components, such a float or internally sensors can compromise the delicate balance required to maintain control preferential crystallization. 


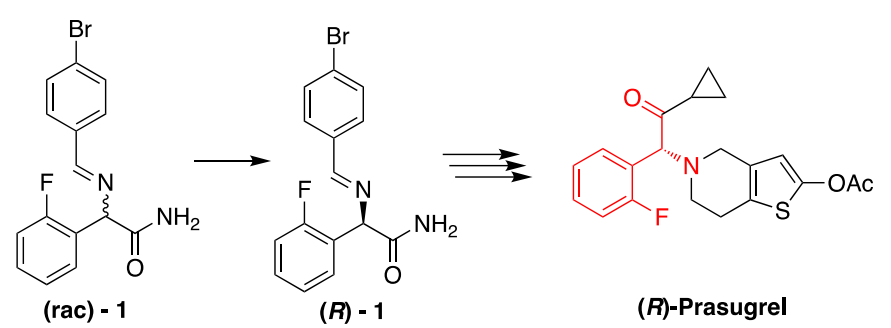

Scheme 1. Resolution of a 2-fluorophenylglycine derivative (1) used to synthesize (R)-Prasugrel.

As a simple proof of concept, racemic 2-flourophenylglycine derivative 1 was selected. This molecule is a well-behaved conglomerate, making it amenable to preferential crystallization. In addition, it provides facile access to the enantiopure amino acid required to synthesize $(R)$-Prasugrel, a compound used for treatment of acute coronary syndrome (Scheme 1). A racemic mixture of 1 was suspended in MeCN recrystallized in a dissolver/crystallizer apparatus using HeinSight to dynamically control the liquid transfer between two $100 \mathrm{~mL}$ reactors. Temperature and stirring were regulated using an EasyMax 102. Chemically resistant tubing was routed through the peristaltic pumps and capped with $9 \mathrm{~mm}$ filter. In this experiment, the webcam was positioned to watch the dissolver (Figure 13 reactor R1). The dissolver was charged with $3.9685 \mathrm{~g}$ of 1 and $80 \mathrm{~mL} \mathrm{MeCN}(0.148 \mathrm{M})$, then stirred at $500 \mathrm{rpm}$. The system was set to a flow rate of $1 \mathrm{~mL} / \mathrm{min}$ and allowed to circulate at $21^{\circ} \mathrm{C}$ for 1.25 hours to ensure equilibrium had been reached. Seeds of crystalline $1(0.1 \mathrm{~g})$ were added to the crystallizer (R2) and the temperature was decreased to $15^{\circ} \mathrm{C}$. The temperature of the dissolver (R1) was maintained at $21^{\circ} \mathrm{C}$. After 7 hours, the solution in the dissolver was transparent indicating that crystallization had reached completion (see Figure $\mathbf{S 1 3}$ in the "CPC" section of the SI). Data from the experimental run is shown in Figure $\mathbf{1 4 .}$

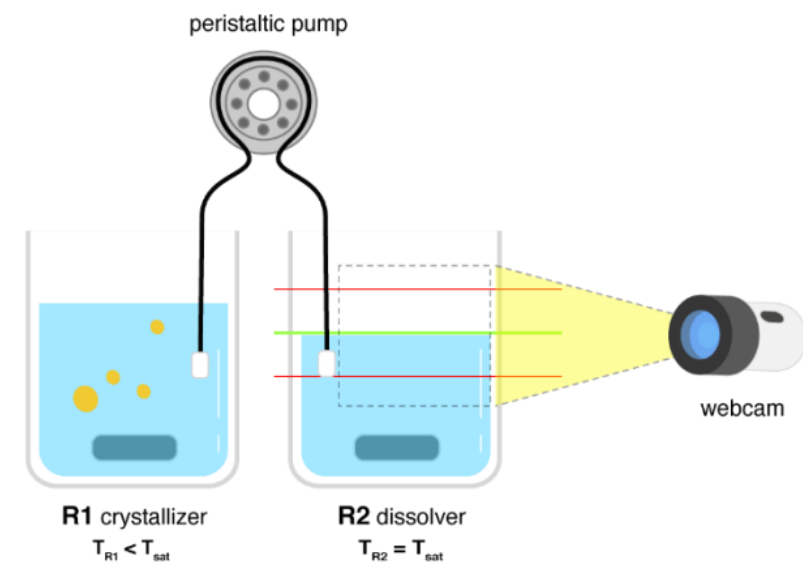

Figure 13. Experimental set-up for a single-pump CPC run.

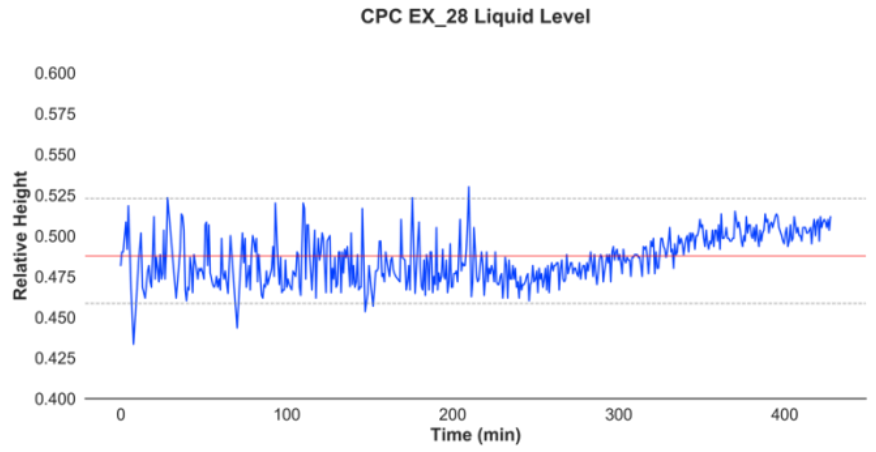

Figure 14. Relative liquid-level height over time during successful CPC of $\mathbf{1}$ in $\mathrm{MeCN}$. Every 8th measurement shown. The red line indicates the reference level; grey dotted lines correspond run specific upper and lower tolerance levels.

\section{Conclusions}

We have developed a generalizable and inexpensive computer-vision based system for liquid-level monitoring and control. It facilitates the autonomation of several common laboratory procedures that typically rely on a human researcher's visual observation. The system can be fully set-up and customized from a GUI and is easily integrated into existing experimental set-ups that require continuous stirring. We have showcased the successful deployment of Heinsight for single and dual-pump CPC, slurry filtration, and evaporative solvent swap experiments.

These applications demonstrate that computer-vision can be used as part of an autonomous platform to monitor experimental factors and make control decisions. More importantly, this study demonstrates how a simple control point, such as maintaining the solvent level in a reactor, can enable multiple, diverse workflows including crystallization, distillation and filtration. Our ability to easily measure and respond to dynamic situational changes are key to enabling and deploying flexible automation workflows.

\section{Conflicts of Interest}

There are no conflicts to declare.

\section{Acknowledgements}

Special thanks to Dr. Joshua Desrap for helping to execute benchtop testing and for troubleshooting prototype software. Thanks to Dr. Céline Rougeot for the synthesis for 2fluorophenylglycine derivatives used in the crystallization proof of concept. We are grateful to the Defense Advanced Research Projects Agency (DARPA) for funding this project under the Accelerated Molecular Discovery Program under Cooperative Agreement No. HR00111920027, dated August 1, 2019. Additional financial support for this work was provided by the University of British Columbia, the Canadian Foundation for Innovation (CFI-35883), and the Natural Sciences and Engineering Resource Council of Canada (RCPIN-2016-04613). 


\section{Notes and references}

(1) Eppel, S.; Kachman, T. Computer Vision-Based Recognition of Liquid Surfaces and Phase Boundaries in Transparent Vessels, with Emphasis on Chemistry Applications. ArXiv14047174 Cs 2014.

(2) Eppel, S. Tracing Liquid Level and Material Boundaries in Transparent Vessels Using the Graph Cut Computer Vision Approach. ArXiv160200177 Cs 2016.

(3) O’Brien, M.; Koos, P.; L. Browne, D.; V. Ley, S. A Prototype Continuous-Flow Liquid-Liquid Extraction System Using Open-Source Technology. Org. Biomol. Chem. 2012, 10 (35), 7031-7036. https://doi.org/10.1039/С2OB25912E.

(4) Kumar, B.; Rajita, G.; Mandal, N. A Review on Capacitive-Type Sensor for Measurement of Height of Liquid Level: Meas. Contro/ 2014. https://doi.org/10.1177/0020294014546943.

(5) Rajamani, A. S.; M, D.; Sai, V. V. R. Plastic Fiber Optic Sensor for Continuous Liquid Level Monitoring. Sens. Actuators Phys. 2019, 296, 192-199. https://doi.org/10.1016/j.sna.2019.07.021.

(6) Ley, S. V.; Ingham, R. J.; O'Brien, M.; Browne, D. L. CameraEnabled Techniques for Organic Synthesis. Beilstein J. Org. Chem. 2013, 9 (1), 1051-1072. https://doi.org/10.3762/bjoc.9.118.

(7) Capitán-Vallvey, L. F.; López-Ruiz, N.; Martínez-Olmos, A.; Erenas, M. M.; Palma, A. J. Recent Developments in Computer Vision-Based Analytical Chemistry: A Tutorial Review. Anal. Chim. Acta 2015, 899, 23-56. https://doi.org/10.1016/j.aca.2015.10.009.

(8) Fitzpatrick, D. E.; Ley, S. V. Engineering Chemistry for the Future of Chemical Synthesis. Tetrahedron 2018, 74 (25), 3087-3100. https://doi.org/10.1016/j.tet.2017.08.050.

(9) Li, J.; Lu, Y.; Xu, Y.; Liu, C.; Tu, Y.; Ye, S.; Liu, H.; Xie, Y.; Qian, H.; Zhu, X. AIR-Chem: Authentic Intelligent Robotics for Chemistry. J. Phys. Chem. A 2018, 122 (46), 9142-9148. https://doi.org/10.1021/acs.jpca.8b10680.

(10) Sprecher, H.; Payán, M. N. P.; Weber, M.; Yilmaz, G.; Wille, G. Acyl Azide Synthesis and Curtius Rearrangements in Microstructured Flow Chemistry Systems. J. Flow Chem. 2012, 2 (1), 20-23. https://doi.org/10.1556/jfchem.2011.00017.

(11) Maslana, E.; Schmitt, R.; Pan, J. A fully automated liquid-liquid extraction system utilizing interface detection https://www.hindawi.com/journals/jamc/2000/864675/ (accessed Mar 27, 2020). https://doi.org/10.1155/S146392460000033X.

(12) Hu, D. X.; O’Brien, M.; Ley, S. V. Continuous Multiple LiquidLiquid Separation: Diazotization of Amino Acids in Flow. Org. Lett. 2012, 14 (16), 4246-4249. https://doi.org/10.1021/ol301930h.

(13) Ingham, R. J.; Battilocchio, C.; Hawkins, J. M.; V. Ley, S. Integration of Enabling Methods for the Automated Flow Preparation of Piperazine-2-Carboxamide. Beilstein J. Org. Chem. 2014, 10, 641-652. https://doi.org/10.3762/bjoc.10.56.

(14) Ingham, R. J.; Battilocchio, C.; Fitzpatrick, D. E.; Sliwinski, E.; Hawkins, J. M.; Ley, S. V. A Systems Approach towards an Intelligent and Self-Controlling Platform for Integrated Continuous Reaction Sequences. Angew. Chem. Int. Ed. 2015, 54 (1), 144-148. https://doi.org/10.1002/anie.201409356.

(15) O'Brien, M.; Cooper, D. A.; Dolan, J. Continuous Flow lodination Using an Automated Computer-Vision Controlled
Liquid-Liquid Extraction System,. Tetrahedron Lett. 2017, 58 (9), 829-834. https://doi.org/10.1016/j.tetlet.2017.01.029.

(16) Rougeot, C.; Hein, J. E. Application of Continuous Preferential Crystallization to Efficiently Access Enantiopure Chemicals. Org. Process Res. Dev. 2015, 19 (12), 1809-1819.

(17) Eppel, S.; Xu, H.; Bismuth, M.; Aspuru-Guzik, A. Computer Vision for Recognition of Materials and Vessels in Chemistry Lab Settings and the Vector-LabPics Dataset. 2020. https://doi.org/10.26434/chemrxiv.11930004.v3.

(18) Schenck, C.; Fox, D. Detection and Tracking of Liquids with Fully Convolutional Networks. ArXiv160606266 Cs 2016.

(19) Mottaghi, R.; Schenck, C.; Fox, D.; Farhadi, A. See the Glass Half Full: Reasoning About Liquid Containers, Their Volume and Content. In 2017 IEEE International Conference on Computer Vision (ICCV); IEEE: Venice, Italy, 2017; pp 18891898. https://doi.org/10.1109/ICCV.2017.207.

(20) Canny, J. A Computational Approach to Edge Detection. IEEE Trans. Pattern Anal. Mach. Intell. 1986, PAMI-8 (6), 679-698. https://doi.org/10.1109/TPAMI.1986.4767851.

(21) Li, Y.-E.; Yang, Y.; Kalthod, V.; Tyler, S. M. Optimization of Solvent Chasing in API Manufacturing Process: Constant Volume Distillation. Org. Process Res. Dev. 2009, 13 (1), 7377. https://doi.org/10.1021/op800152n.

(22) Elgue, S.; Prat, L.; Cabassud, M.; Cézerac, J. Optimisation of Solvent Replacement Procedures According to Economic and Environmental Criteria. Chem. Eng. J. 2006, 117 (2), 169-177. https://doi.org/10.1016/j.cej.2005.11.017.

(23) Levilain, G.; Coquerel, G. Pitfalls and Rewards of Preferential Crystallization. CrystEngComm 2010, 12 (7), 1983-1992. https://doi.org/10.1039/C001895C.

(24) Lorenz, H.; Seidel-Morgenstern, A. Processes To Separate Enantiomers. Angew. Chem. Int. Ed. 2014, 53 (5), 1218-1250. https://doi.org/10.1002/anie.201302823.

(25) Hein, J. E.; Cao, B. H.; van der Meijden, M. W.; Leeman, M.; Kellogg, R. M. Resolution of Omeprazole Using Coupled Preferential Crystallization: Efficient Separation of a Nonracemizable Conglomerate Salt under Near-Equilibrium Conditions. Org. Process Res. Dev. 2013, 17 (6), 946-950. https://doi.org/10.1021/op400081c.

(26) Dunn, A. S.; Svoboda, V.; Sefcik, J.; ter Horst, J. H. Resolution Control in a Continuous Preferential Crystallization Process. Org. Process Res. Dev. 2019, 23 (9), 2031-2041. https://doi.org/10.1021/acs.oprd.9b00275.

(27) Binev, D.; Seidel-Morgenstern, A.; Lorenz, H. Continuous Separation of Isomers in Fluidized Bed Crystallizers. Cryst. Growth Des. 2016, 16 (3), 1409-1419. https://doi.org/10.1021/acs.cgd.5b01513.

(28) Geyyer, R.; Dürr, R.; Temmel, E.; Li, T.; Lorenz, H.; Palis, S.; Seidel-Morgenstern, A.; Kienle, A. Control of Continuous Mixed-Solution Mixed-Product Removal Crystallization Processes. Chem. Eng. Technol. 2017, 40 (7), 1362-1369. https://doi.org/10.1002/ceat.201600692. 